\title{
UBE2V2 promotes metastasis by regulating EMT and predicts a poor prognosis in lung adenocarcinoma.
}

\section{Zheng Yang}

Affiliated Hospital of Nantong University

\section{Qiang Xue}

Affiliated Hospital of Nantong University

Caishen Du

Affiliated Hospital of Nantong University

Miaosen Zhen

Affiliated Hospital of Nantong University

Jianxun Xu

Affiliated Hospital of Nantong University

Pengfei Zhang

Affiliated Hospital of Nantong University

Jianmei Zhao

Affiliated Hospital of Nantong University

Guanglin Shi

Affiliated Hospital of Nantong University

Xianchen Liu

Affiliated Hospital of Nantong University

Zhiyuan Tang

Affiliated Hospital of Nantong University

Xiaoyu Zhou ( $\nabla 60729468 @ q q . c o m$ )

Affiliated Hospital of Nantong University https://orcid.org/0000-0002-3786-7690

\section{Primary research}

Keywords: Apoptosis, Bioinformatic, EMT, LUAD, Proliferation, UBE2V2

Posted Date: March 18th, 2021

DOI: https://doi.org/10.21203/rs.3.rs-316728/v1 
License: (c) (i) This work is licensed under a Creative Commons Attribution 4.0 International License. Read Full License 


\section{Abstract}

Background: Belonging to the ubiquitin-conjugating enzymes (E2s) family, UBE2V2 demonstrated a remarkable tumourigenic ability in many kinds of cancers. However, the interrelationship between UBE2V2 expression and morbidity of lung adenocarcinoma (LUAD) is still unknown.

Methods: By using TCGA predictions and clinical tissue samples, we assessed the expression of UBE2V2 mRNA and protein in LUAD and analyzed its relationship with different clinicopathological factors as well as survival prognosis. Besides, we further studied the EMT signaling pathway that promotes LUAD metastasis and other phenotypic experiments by using lentivirus to transfect LUAD cells.

Results: Our research results showed that compared with normal tissues, the expression of UBE2V2 mRNA and protein in LUAD was significantly increased $(P<0.001)$. UBE2V2 might be considered as an independent prognostic molecule for LUAD patient survival prognosis based on TCGA prediction (HR:1.497 P=0.012) and immunohistochemical analysis (ICH) (HR:1.842 P=0.042). ICH showed that UBE2V2 was related to the following clinicopathological factors, including gender $(P=0.021)$, stage $(P=0.042)$, lymph node metastasis $(P=0.021)$ and differentiated degree $(P=0.015)$. Finally, knockdown of UBE2V2 significantly reduced the migration ability by regulating the EMT pathway. The knockdown of UBE2V2 inhibited cells proliferation, reduced the proportion of cells in S phase and promoted cell apoptosis. Interestingly, UBE2V2 expression is negatively correlated with B cells, CD4 + T cells, macrophages and dendritic cells.

Conclusions: In summary, UBE2V2 might play an important role in the progression of LUAD.

\section{Introduction}

Lung cancer poses a threat in male mortality, which is second to breast cancer for women [1]. On the basis of histopathology, lung cancer is composed of two types: Non-Small Cell Lung Cancer (NSCLC) and Small Cell Lung Cancer (SCLC). Among on the lung cancers, NSCLC is about $80 \%$ and mainly constitutes lung adenocarcinoma (LUAD) and lung squamous cell carcinoma (LUSC)[2]. LUAD is the most common histological subtype based on the clinicopathological diagnosis[3]. At the time of diagnosis, LUAD was in a locally advanced or metastatic stage, leaving no time for early detection or treatment[4]. In recent years, despite having more diagnostic technologies and novel treatment methods, a large number of LUAD patients were still diagnosed as advanced stage and were resistant to traditional chemotherapy drugs so that their 5-year overall survival (OS) remains low[5]. According to clinical practice and basic research, the early metastasis of lung cancer often results in a higher degree of malignancy and poor treatment effect. Hence, to raise the long-term survival prognosis of LUAD patients, it is essential to discover an effective molecular biomarker, which will be used for the early diagnosis and treatment of LUAD. 
Epithelial-mesenchymal transition (EMT) refers to the biological process of epithelial cells transforming into cells with mesenchymal phenotype through different signal pathways under adverse factors, which allows these cells to gain capability of infiltration and invasion and migration[6]. EMT involves the following steps in tumour metastasis: firstly, epithelial tumour cells degrade the basement membrane and invade surrounding parenchymal tissues (invasion), and then epithelial tumour cells pass through endothelial cells into blood vessels and lymphatic channels (intravascular infiltration). Furthermore, tumour cells metastasize to various parts of the body under the entire body fluid circulation system (systemic transport). Eventually, tumour cells surviving in the blood pass through capillaries into the distant parenchymal organs (extravasation) and continue to grow malignant tumours in the new matrix environment (colonization) [7-9]. The fundamental characteristic of EMT is the decreased expression of Ecadherin and upregulated expression of Vimentin and N-cadherin[10]. In the process of morphological changes of neuroectoderm and mesoderm cells, Snail promotes the conversion of DE-cadherin expression to DN- cadherin type so that EMT occurs[11].In addition, EMT transformation promoted the expression of MMPs family proteins (MMP 1, 2,7) through the up-regulated expression of Snail and SIP1, and degraded extracellular matrix proteins to increase tumour cell infiltration ability[12]. Pan et al. [13] study discovered that up-regulation of MMP2/MMP9 expression in cholangiocarcinoma induced epithelial-mesenchymal transition (EMT), which led to lymph node metastasis. Wu D M et al. [14] showed that the Inc-MMP-2 increased the ability of migration and invasion by promoting the expression of MMP2 in lung cancer. In lung cancer, EMT is often a huge connection with poor prognosis, including increased tumour invasion and distant metastasis, enhanced resistance to chemotherapy drugs and led to the development of lung cancer stem cells[15].

Recently, many studies have found that the ubiquitin-protease system (UPS) was related to the occurrence of many malignant tumours. Ubiquitylation is mediated via a suit of enzymes. Firstly, ubiquitin (Ub)-activating enzymes (E1) hydrolyzes ATP to form high-energy thioesters between the $\mathrm{COOH}$ terminus of $\mathrm{Ub}$ and the cysteine of E1. The activated ubiquitin was delivered to the ubiquitin -conjugating enzymes (E2) and transferred diametrically to the substrate protein or the ubiquitin-ligase enzymes(E3) [16]. Ubiquitin-conjugating E2 enzyme variants (UBE2V) belongs to the ubiquitin-family and contains two family members, UBE2V1 and UBE2V2[17, 18]. UBE2V2 (otherwise called hMMS2; DDVIT1; EDAF-1) is essential for genome maintenance in the nucleus through DNA damage repair pathways[19].

Overexpression of UBE2V2 induced the occurrence of many types of tumours, for instance Melanoma[20], colorectal carcinoma[21], breast cancer[22]. However, the mechanism and function of UBE2V2 in LUAD prognosis remains enigmatic. In this report, we jointly analyzed the mRNA and protein level of UBE2V2 in LUAD and normal samples in light of the prediction of TCGA biometrics analysis, ICH analysis and Western blotting. Meanwhile, we made analysis of the relationship between UBE2V2 and clinicopathological parameters and survival prognosis. Furthermore, we studied in vitro whether knockdown UBE2V2 affected the invasion, migration, proliferation and apoptosis of LUAD. The results indicated that the invasive capability of LUAD was remarkably reduced after knockdown UBE2V2, which was through the inhibition of EMT signal pathway. Beside, knockdown UBE2V2 inhibited the proliferation 
of LUAD cells and increased the proportion of apoptosis. Interestingly, we also found that UBE2V2 regulated the level of immune cell infiltration in LUAD.

\section{Materials And Methods}

\section{Collection of RNA-sequencing data based on the TCGA database}

LUAD samples mRNA (551 samples in total, including 54 normal samples) and matched clinical characteristic parameters were acquired from TCGA (https://portal.gdc.cancer.gov/). The TCGA uses large-scale high-throughput genome sequencing analysis technology to assist humans better understand tumors, thereby improving cancer prevention, diagnosis and treatment capabilities. The Illumina HiSeq_RNA-Seq platform provided all LUAD sequencing data, which was used to analyze the role of UBE2V2 in LUAD. After removing samples with incomplete survival information and lost follow-up from TCGA-LUAD, COX regression analyzed 465 samples with entire clinical information. Based on the TCGA, Strawberry Perl and R (3.5.2) software was used for bioinformatics analysis.

\section{Tissue samples of patients}

Ten pairs of fresh cancer and adjacent tissues from patients who had not received any radiotherapy or chemotherapy were collected for Western blotting. Between 2013 and 2020, specimens of 91 LUAD patients who had not received any radiotherapy or chemotherapy before undergoing surgery at Nantong University Affiliated Hospital were collected for $\mathrm{ICH}$ analysis. The patients participating in this study were informed that their tissues and related clinical information would contribute to scientific research, and signed an informed consent form. The Ethics Committee of Nantong University Affiliated Hospital had agreed to the application of these specimens and data in scientific research.

\section{Immunohistochemistry}

We fixed the fresh tissue samples with $10 \%$ formalin before embedding. Then these paraffins embedded tissue samples were made into four micron-thick tissue slices. After 6 hours of baking in a $60{ }^{\circ} \mathrm{C}$ incubator, the tissue sections were immersed in ethanol of different concentrations for dewaxing. In order to block the activity of endogenous peroxidase, $3 \%$ hydrogen peroxide $(\mathrm{H} 2 \mathrm{O} 2)$ was dropped on the surface of the tissue sections, at $121^{\circ} \mathrm{C}$. The tissue sections incubated with anti-UBE2V2 polyclonal antibody (dilution $1: 100$ ) at $25^{\circ} \mathrm{C}$ for 2 hours. Using PBS solution to wash the tissue sections 3 times, each for 10 minutes. The secondary antibody matched with anti-UBE2V2 polyclonal antibody was dripped onto the surface of the tissue section at $25^{\circ} \mathrm{C}$ for 1 hours. The DAB solution was dripped onto the surface of the tissue section to catalyze the formation of a water-insoluble brown-yellow product for visualization. After being repeatedly washed by water, the tissue sections were stained with hematoxylin, then the sections dripping with neutral resin were sealed with cover glass. The staining results of the 
tissue section were evaluated by three experienced pathologists. The proportion of stained cells in the tissue section was scored based on the following score: 1 (1-30\%), 2 (31- 50\%), $3(51-70 \%)$, or 4 (71$100 \%$ ). The staining intensity of the tissue sections was scored based on the following criteria: 0 (no staining), 1 (light yellow), 2 (deep yellow), and 3 (brown particles). According to the multiplication size of two scores, UBE2V2 expression was divided into high and low score groups: low score group was from 14 , and high score group was from 6-12.

\section{Western blotting}

The crushed fresh LUAD tissue and cells cultured for 48 hours were added to the lysis buffer to collect protein. After centrifugation at 2,0000 $\mathrm{xg}$ for one quarter of an hour, the protein-containing supernatant was transferred to the enzyme-free centrifuge tube. BioRad protein assay was used to quantify protein concentration (BioRad Laboratories, Inc). At a voltage of $120 \mathrm{~V}$, SDS-PAGE gels were used to separate proteins from different samples. Then, the protein on the gel was transferred to the PVDF (polyvinylidene fluoride) microporous membrane at electric current of $200 \mathrm{~mA}$. The PVDF membrane containing protein was soaked in $5 \%$ skim milk at $25^{\circ} \mathrm{C}$ for 2 hours. The diluted primary antibody was added to the blocked PVDF membrane and incubated at $4^{\circ} \mathrm{C}$ for 12 hours. TBST washed the membrane 3 times, 20 minutes each time. The HRP-conjugated secondary antibody (dilution 1:10000, cat. no. A0208; Beyotime Institute of Biotechnology) was added to the PVDF membrane containing the protein and incubated for 2 hours at $25^{\circ} \mathrm{C}$. Similarly, TBST washed the PVDF membrane 3 times, 20 minutes each time. Primary antibody antiUBE2V2 (D624139-0001) was purchased from Sangon Biotech. Anti-GAPHD (10494-1-AP), anti-MMP2 (10373-2-AP), anti-Vimentin (10366-1-AP), anti-E-cadherin (20874-1-AP), anti-PCNA (10205-2-AP) and anti-N-cadherin (22018-1-AP) were acquired from Proteintech. Finally, the protein bands separated by gel electrophoresis were recognized by the ECL detection systems (Pierce, Rockford, IL, USA).

\section{Cell culture and transfection}

In present study, four LUAD cell lines including H1299, A549, SPCA1 and H1650 were acquired from the Shanghai Institute of Cell Biology Academia. Then these LUAD cells were fostered in RPMI 1640 medium containing $10 \%$ fetal bovine serum (FBS). Cancer cells in logarithmic growth were generally used in experiments from the third to sixth generation. For UBE2V2 silencing, three different UBE2V2-shRNAs lentiviruses and one Con-shRNA lentiviruses were designed via GeneChem Technologies: primer sequence $\left(5^{\prime}-3^{\prime}\right)$ of shRNA-1 GCCCGGAGCATACCAGTGTTA; primer sequence (5'-3') of shRNA-2

CAAGGTGGACAGGCATGATTA; primer sequence (5'-3') of shRNA-3 GTCTTAAATCAACAACCTTCT; and primer sequence (5'-3') of Con077 (shCon) TTCTCCGAACGTGTCACGT. A549 and SPCA1 cells were selected for lentiviral transfection. After 24 hours, the cell culture medium was replaced, and stable UBE2V2-shRNA expression cells were obtained after selection with puromycin. The operation of the above experiment, we strictly followed the manufacturer's reference guide. 


\section{Cell migration assays}

The collected $2 \times 10^{4}$ cells were transferred into the Transwell chamber in a 24-well plate. $500 \mu \mathrm{l}$ of medium with FBS was put into the lower chamber of the 24-well plate to facilitate cell migration. After 48 hours, methanol was used to fix the cells that migrate to the lower chamber of the 24-well plate for 20 minutes, and $0.1 \%$ crystal violet staining was used for cell staining for half an hour. A microscope (Olympus, Japan) with a 40x objective lens was used to take photos and record the migrated cells.

\section{Cell cycle analysis}

After collecting the cells in logarithmic growth in the 6-well plates, pre-cooled PBS was used to wash the cells twice (add $3 \mathrm{ml}$, suspend, and centrifuge), and then the collected cells were fixed in pre-cooled $75 \%$ absolute ethanol at $4^{\circ} \mathrm{C}$ overnight. 500uLPBS containing $50 \mathrm{ug} / \mathrm{mL}$ ethidium bromide (PI), $100 \mathrm{ug} / \mathrm{mL}$ RNase $\mathrm{A}, 0.2 \%$ Triton $\mathrm{X}-100$ was used to incubate the cells for half an hour at $4^{\circ} \mathrm{C}$ in the dark. The processed cells were identified by Attune NxT flow cytometry whether they were in G1, S or G2/M phase.

\section{Apoptosis analysis}

The adherent shCon and shUBE2V2 cells $\left(1 \times 10^{6} / \mathrm{mL}\right)$ were trypsinized and collected in a $10 \mathrm{ml}$ centrifuge tube. These cells were rinsed twice with pre cooled PBS. Immediately afterwards, these cells were centrifuged at $4{ }^{\circ} \mathrm{C}$ for 5 minutes at $2000 \mathrm{~g}$. Then 5 uLAnnexin $\mathrm{V}$ and $5 \mathrm{uL}$ PI labeled with fluorescent dyes were added to the cells and incubated in the dark at $25^{\circ} \mathrm{C}$ for 15 minutes. Finally, flow cytometry (FACSCalibur, Becton-Dickinson, USA) was used to detect cell apoptosis.

\section{Colony formation assay}

Cells in logarithmic division were transferred into six-well plate (800 cells/well). After 10 days of cultivation, the cloned cells were immersed in methanol for fixation for 30 minutes, and stained with Giemsa solution for a quarter of an hour. The number of colonies was recorded by fluorescence microscope (Olympus). The colony forming assay was repeated three times in duplicate.

\section{Gene set enrichment analysis of UBE2V2}


GSEA is a calculation measure used to explain that a gene shows a remarked statistical difference between the high and low groups. We executed GSEA on the normalized RNA-Seq data obtained from TCGA[23]. We uploaded the generated data set and phenotype label file to the GSEA software. After the phenotype was marked as UBE2V2 high and UBE2V2 low, the KEGG pathway of UBE2V2 in GSEA was acquired. $\mathrm{P}<0.05$ and $\mathrm{FDR}<0.05$ was regarded as enrichment.

\section{The correlation between UBE2V2 and Immune Infiltrating cells}

The "Gene" module of TIMER was used to investigate the association between UBE2V2 and a variety of immune cells (CD4 + T cells, dendritic cells, B cells, CD4 + T cells, B cells, neutrophils, and macrophages) and tumor purity in LUAD. TIMER (https://cistrome.shinyapps.io/ timer/) systematically analyzed the immune infiltration of various cancer.

\section{Statistical Analysis}

We applied GraphPad Prism8.0 and SPSS 24.0 to statistics respectively. We applied unpaired student's ttest to the comparison of two groups. We applied Kaplan-Meier method to predict survival rate of patients. $\mathrm{P}<0.05$ was regarded as a significant difference. All experiments in this study were repeated three times.

\section{Results}

\section{UBE2V2 was up-regulated in LUAD tissues compared to normal samples}

The 497 LUAD and 54 normal samples obtained from the TCGA network platform were analyzed by R ( $v$ 3.5.2). We compared the mRNA of UBE2V2 between LUAD and adjacent normal samples. The result predicted that the UBE2V2 mRNA in LUAD was remarkably higher than normal tissues $(P<0.001)$ (Fig. 1a, b). To further validate the above result of the TCGA, the protein level of UBE2V2 was tested by Western blotting in 10 pairs of fresh LUAD and normal adjacent tissues. The results revealed UBE2V2 protein expression was remarkably up-regulated in LUAD (Fig. 1C). We inferred that UBE2V2 might participate in the development of LUAD.

\section{Correlations between expression of UBE2V2 and clinical parameters}

To begin with, by using the "ggplot2" package to explore that overexpression of UBE2V2 was obviously associated with Gender $(P=0.0031$, Age $(P=0.0019)$ and T classification $(P=0.041)$ based on TCGA (Fig. 2a-f). Then, ICH analysis revealed the association between UBE2V2 expression and clinicopathological characteristics. The low level of UBE2V2 expression was 41 samples (45.05\%) in 
total, and the other $50(54.95 \%)$ samples showed high level of UBE2V2 expression. The result showed that UBE2V2 was correlated with four factors: Gender ( $P=0.021)$, Tumour Stage $(P=0.042)$, Differentiated degree $(P=0.015)$ and Lymph node metastasis $(P=0.021)$ (Table 1$)$.

\section{UBE2V2 predicted poor overall survival in LUAD patients}

In TCGA, "survival" package was used to draw survival curves. The overall survival (OS) analysis illustrated that high UBE2V2 expression had a worse prognosis than low UBE2V2 expression in LUAD patients ( $P=0.047)$ (Fig.3a). Through GEPIA, the same survival curve trend was proven $(P<0.001)$ (Fig. $3 b)$. The Percent Survival of ICH also verified the above results: high expression of UBE2V2 tended to have a worse prognosis in LUAD ( $P=0.0132)$ (Fig. 3c). Meanwhile, the univariate analysis based on TCGA forecasted that Stage (HR: 1.65; 95\% Cl: 1.42-1.92; P<0.01), T classification (HR: 1.53; 95\% Cl: 1.25-1.87; $\mathrm{P}<0.01)$, N classification (HR: 1.69; 95\% Cl: 1.41-2.03; $\mathrm{P}<0.01$ ), and UBE2V2 expression (HR:1.46; 95\%Cl: 1.07-2; $P=0.014$ ) were remarkably related with overall survival (Table 2). And the multivariate analysis, which depicted as a forest boxplot, forecasted that UBE2V2 expression $(P=0.012)$ (Fig. 3e) was an independent prognosis factor for LUAD. The same results were verified via ICH. In the ICH, univariate analysis discovered the up-regulation of UBE2V2 especially affected the survival of LUAD patients in all variables (HR: 2.002, 95\% Cl: 1.139-3.517, $\mathrm{P}=0.016$ ) (Table 3). And multivariate analysis indicated UBE2V2 (HR: $1.816,95 \% \mathrm{Cl}: 1.046-3.315, \mathrm{P}=0.042$ ) could be considered as an independent prognostic factor in LUAD (Table 3). In summary, UBE2V2 might be predicted as an adverse molecular marker for malignant progression of LUAD based on the TCGA and ICH analysis.

\section{Diagnostic value of UBE2V2 expression in LUAD}

So as to evaluate whether UBE2V2 had diagnostic value in LUAD, 497 tumour samples and 54 normal samples from the TCGA database were used to generate receiver operating characteristic (ROC) curves. The "pROC" package was used to calculate the area under the curve, which is 0.772 . This analysis indicated that UBE2V2 had a considerable diagnostic value in LUAD (Fig. 3d).

\section{LUAD cell lines selection and transfection efficiency}

For further molecular mechanism and phenotype research, two LUAD cell lines were selected from four LUAD cell lines to apply to the next experiment. The UBE2V2 protein expression levels in A549 and SPCA1 cells were relatively high compared to $\mathrm{H} 1299$ and $\mathrm{H} 1650$ cells via Western blotting (Fig. 4a, b). Therefore, A549 and SPCA1 cells were selected to be transfected with UBE2V2-shRNAs and Con-shRNA lentiviruses. Western blotting detected the transfection efficiency of lentivirus. The results showed UBE2V2 expression 
was down-regulated with three different shUBE2V2 primer sequences, and the expression of UBE2V2 was most significantly decreased in shRNA-2 (Fig. 5 a, b and 6 a, b).

\section{Knockdown of UBE2V2 suppressed the migration in A549 and SPCA1 cells}

We applied Transwell analysis to investigate whether knockdown of UBE2V2 affected the migration ability of LUAD cells. The results demonstrated that knockdown of UBE2V2 (especially shRNA-2) remarkably inhibited the migration of A549 and SPCA1 cells from the upper chamber to the lower chamber in the 24-well plate (Fig. $5 \mathrm{a}, \mathrm{g}$ and $6 \mathrm{a}, \mathrm{g}$ ).

\section{Knockdown of UBE2V2 inhibited EMT process in SPCA1 and A549 cells}

As we known, tumour metastasis involves many replication processes, including the loss of polarity on the top-bottom surface of epithelial cells, the absence of tight junctions and adhesion junctions between cells and the degradation of extracellular matrix and basement membrane. To investigate whether UBE2V2 was related to epithelial-mesenchymal transition (EMT) in LUAD, EMT-related proteins including E-cadherin, N-cadherin, Vimentin and MMP2 were studied via Western blotting. The results showed that the expression of cell epithelial protein E-cadherin protein was significantly increased, while the expression of proteins with characteristics of mesenchymal cells such as Vimentin, N-cadherin and MMP2 protein was obviously reduced after Knockdown of UBE2V2 (especially shRNA-2) in A549 and SPCA1 cells (Fig. 7a and 8a).

\section{Knockdown of UBE2V2 suppressed the proliferation and induced apoptosis in A549 and SPCA1 cells}

In order to explore whether UBE2V2 affected the proliferation and apoptosis of LUAD, a series of experiments were used in our research. Colony formation assay showed that the number of colony cells with knockdown of UBE2V2 were clearly reduced compared to shCon cells (Fig. 7a, b). Through flow cytometry analysis, we detected the proportion of healthy cells, early/late apoptotic cells and necrotic cells in knockdown of UBE2V2 cells. The results observed a remarkable increase in the percentage of early/late apoptotic cells (especially shRNA-2) in A549 and SPCA1 cells compared to shCon cells (Fig.7c, d). Western blotting analysis displayed that the proliferation marker PCNA remarkably reduced in LUAD cells after knockdown of UBE2V2 (Fig. 4 c, d, e).

\section{Knockdown of UBE2V2 led to cell-cycle arrest in A549 and SPCA1 cells}


Flow cytometry was used to analyze whether knockdown of UBE2V2 affects the cell cycle in LUAD cells. After knockdown of UBE2V2, cell replication stagnated in the early stage of DNA synthesis (G1 phase): the proportion in the GI phase increased significantly, yet the proportion was remarkably diminished in the DNA synthesis phase (S phase) (Fig.7e, 7f).

\section{GSEA investigation of UBE2V2}

The signaling pathway of UBE2V2 involved in the development of LUAD was obtained by GSEA. GSEA showed remarkably differences (P-value $<0.050$, FDR $<0.050$ ) though enrichment of KEGG pathways in sample high expression of UBE2V2. Based on the normalized enrichment score (NES), the enrichment signaling pathway with the most significant phenotypic enrichment was selected. KEGG pathway analysis identified five pathways that had a positive correlation with UBE2V2 expression: DNA replication, cell cycle, mismatch repair, ubiquitin mediated proteolysis and nucleotide excision repair. The five pathways with the strongest negative correlation were arachidonic acid metabolism, allograft rejection, hematopoietic cell lineage, leukocyte trans endothelial migration, intestinal immune network for IgA production, as shown in Fig. 8a. These results indicated that the pathways regulating DNA damage repair and immune microenvironment, which are critically important in LUAD patients.

\section{The association between UBE2V2 and TIICs in LUAD}

The association between UBE2V2 expression and the level of infiltration of different immune cells was studied through the "Gene" panel of TIMER (Fig. 8b). The results revealed that UBE2V2 was positively bound up with tumour purity in $\operatorname{LUAD}(r=0.105, P=0.019)$ and $C D 8+T$ cells $(r=0.095, P=0.036)$, but negatively bound up with $B$ cells $(r=-0.245, P<0.0001), C D 4+T$ cells $(r=-0.238, P<0.0001)$, macrophages $(r=-0.106, P=0.019)$, and dendritic cells $(r=-0.188, P<0.0001)$.

\section{Discussion}

UBE2V2, a ubiquitin-conjugating enzyme variant protein, belongs to a subfamily of E2 protein family[24]. UBE2V2 lacking the active site of cysteine binds to specific UBC13 to form a heterodimer, which catalyzes ubiquitin to arrange a K63-linked non-canonical polyubiquitin chain[17, 18, 25]. The special structural features of UBE2V2 may result in different functions in cancer from other classic E2 enzymes.

In this report, mRNA of UBE2V2 was highly expressed in LUAD, which was predicted by the bioinformatics analysis of the TCGA database. IHC and Western blotting results also suggested UBE2V2 protein level in LUAD samples were higher than normal samples. The low expression UBE2V2 had a better survival prognosis than the high expression UBE2V2 in LUAD, which was first forecasted by the TCGA and GEPIA databases. The same Kaplan-Meier survival curve result was verified via the ICH. Multivariate Cox analyses of both ICH and TCGA databases indicated that the UBE2V2 expression was a potential 
independent marker for poor prognosis in LUAD. These results strongly indicated that UBE2V2 might be involved in the development of LUAD. The result of ICH suggested that UBE2V2 was related to lymph node metastasis. Based on the above-mentioned key research clue, we tested whether UBV2V2 at the cellular level was related to the migration of LUAD through Transwell assay. Transwell assay manifested that knockdown UBE2V2 inhibited the migration ability of LUAD. The result was consistent with the clinical data study, which indicates that UBE2V2 might affect the invasion and migration ability of LUAD cells. However, there are few studies involving the relationship between UBE2V2 and the invasion and migration of malignant tumors. Only found in melanoma, inhibiting the expression of UBE2V2 upregulated the expression of E-Cadherin (an EMT marker), but the specific mechanism has not been further studied[20]. In colorectal cancer, UBE2V1 (another family member of UBE2Vs) promotes the epithelialmesenchymal transition (EMT) and distant invasion and metastasis of colorectal cancer cells by inhibiting the occurrence of autophagy[26]. Therefore, we speculated whether UBE2V2 also promotes the metastasis of LUAD by regulating EMT singnal pathway.

It's widely known that epithelial mesenchymal transition is bound up with the invasion and metastasis of many cancers, namely EMT[27]. Most LUAD patients tend to have distant metastasis when they are first diagnosed. Therefore, it is difficult to determine at which stage EMT occurs in LUAD based on the current level of diagnosis and treatment[28]. LUAD patients with EMT regulate many biological changes, of which the most obvious and important is the existence of drug resistance[29]. Our above research results indicated that UBE2V2 was related to metastasis of LUAD. However, it is unclear whether UBE2V2 increases the ability of invasion and metastasis in LUAD through EMT. Thence, the correlation between knockdown UBE2V2 and EMT-related proteins was further explored. Our data indicated that knockdown UBE2V2 increased the expression of E-cadherin, while decreased the expression of Vimentin, $\mathrm{N}$-cadherin and MMP2. The above experimental results revealed knockdown UBE2V2 inhibited metastasis of LUAD cells via regulating EMT signal pathway. The specific mechanism of UBE2V2 resulting in tumourigenesis EMT remains further study.

Moreover, UBE2V2, as the first to be discovered, playing an important role in DNA damage repair in eukaryotes[19]. UBC13-UBE2V2 interacted with the DNA binding protein RAD5 (an E3 ligase) and was recruited to the chromatin[30, 31]. The polyubiquitin chain formed by the RAD5-UBE2V2-UBC13 complex in the chromatin mediates the recombination repair after DNA replication by an error-free way of template conversion[32]. In organisms, DNA is extremely susceptible to be damaged by various external and internal factors. DNA damage repair system is highly conserved from yeast to humans, including nuclear excision repair, base excision repair, photo resurrection, mismatch repair, double-strand break repair, and cross-damage replication, etc[33]. When DNA was damaged, UBC13-UBE2V2 and RAD5 proteins could form a K63-linked polyubiquitin chain and performed polyubiquitination modification on the PCNA at the primer-template junction through an error-free lesion bypass way to avoid DNA damage[34-36]. The 
polyubiquitinated PCNA exposes the IDCL (interdomain connector loop, IDCL) domain, which made the DNA polymerase Poln and other $Y$ family polymerases closed to PCNA, leading to the conversion of polymerases and played a huge role in DNA damage repair[37]. In the study, KEGG pathway analysis predicted that UBE2V2 was mainly positive related to cell cycle, nucleotide excision repair, mismatch repair, and DNA replication. Santarpia et al.[22] study showed that the high expression of UBE2V2 in breast cancer can protect tumor cells from DNA damage induced by chemotherapy through DNA repair pathways. Dikshit A et al.[20] showed that silencing UBC13 and UBE2V2 markedly reduced melanoma cells proliferation by activating MEK/FRA1/SOX10 pathway. Here, our data revealed that knockdown UBE2V2 remarkably restrained the proliferation of A549 and SPCA1 cells by Colony formation assay. A remarkably lessen in the percentage of $S$ phase and a tremendously improve in the percentage of $\mathrm{G} 1$ phase was found after knockdown of UBE2V2 in SPCA1 and A549 cells. Western blotting showed knockdown of UBE2V2 significantly inhibited PCNA expression in SPCA1 and A549 cells. Therefore, for the previous researches mentioned above and the discoveries of this experiment, we speculated that the UBE2V2 in lung cancer would increase the polyubiquitination of PCNA in the S phase, which would induce the transfer of damaged DNA from the replication mechanism to the trans-injury synthesis pathway mediated by RAD5-UBE2V2-UBC13 protein, and eventually led to lung cancer cells to develop tolerance to a variety of targeted therapies and immune drugs.

The immune infiltration of tumours has attracted attention in recent years. The tumour microenvironment (TME) is one of the important factors leading to the malignant progression of tumours[38]. It has been found in many tumour models that $C D+4$ and $C D+8 T$ cells could inhibit tumour growth and caused the regression of solid tumours[39-41]. CD4 + T cells cause tumour regression through an IFN-c-dependent mechanism, which is by recruiting macrophages and/or eosinophils[42]. We used the TIMER database to reveal the link between UBE2V2 and different types of immune cell infiltration levels in LUAD. Our data revealed UBE2V2 was significantly negatively associated with CD4+T cells, Macrophages and B cells. This suggests overexpression of UBE2V2 inhibits the infiltration of immune cells in LUAD, which may also provide us with a new idea for immunotherapy.

To sum up, at the beginning we predicted the expression pattern of UBE2V2 in LUAD through the biological information analysis based on the TCGA database and verified the prediction results through $\mathrm{ICH}$ and in vitro cell experiments. Our data indicated that UBE2V2 was overexpression in LUAD and could be used as an independent prognostic indicator of LUAD. Secondly, we studied the function and mechanism of UBE2V2 in LUAD through in vitro experiments. The results showed that UBE2V2 caused invasion and metastasis of A549 and SPCA1 cells via activating EMT signal pathway. Meanwhile, the experimental results also revealed that UBE2V2 could increase the proliferation capacity and reduced the apoptosis of A549 and SPCA1 cells. In summary, UBE2V2 might play an important role in the progression of LUAD. However, our experiments were only limited to TCGA predictions and in vitro studies. Although 
we tried to expand the sample size of experimental data, it will inevitably be affected by sample size and selection bias. Therefore, we will try to establish a UBE2V2-deletion mouse model to further analyze the effects of UBE2V2 on the metastasis, proliferation and apoptosis of LUAD so as to improve the reliability of the experiment. This will exert a profound impact on the further application of our experimental results in clinical practice.

\section{Abbreviations}

UBE2V2: Ubiquitin-conjugating E2 enzyme variants 2; LUAD: Lung Adenocarcinoma; LUSC: Lung Squamous Cell Carcinoma; EMT: Epithelial-mesenchymal transition; TCGA: The Cancer Genome Atlas; 95\% Cl: 95\% confidence interval; HR: Hazard ratio; GSEA: Gene Set Enrichment Analysis; RIPA: Radio Immunoprecipitation Assay; ICH: Immunohistochemistry; PVDF: Polyvinylidene fluoride; TBST: TrisBuffered Saline Tween; FBS: Fetal bovine serum.

\section{Declarations}

\section{Acknowledgements}

Thank you to patients and their families for agreeing to use tissue samples and clinical information. We thank Dr. Xiaoyu Zhou and Ms. Zhiyuan Tang for carefully reading the manuscript.

\section{Authors' contributions}

$X Y, Z$ and $Z Y, T$ contributed to study design; $Z Y$ and $Q X$ contributed to manuscript editing and experimental studies; MS,Z PF,Z CS,D and JX,X: data management and data collection. JM,Z and GL,S contributed to data analysis. All authors read and approved the final manuscript.

\section{Funding}

This work was supported by Nantong Science and technology plan (No. MS22019001; HS2018003), The Clinical Frontier Key Project of Jiangsu Province (No.BE2018670) and Nantong Science and Technology Plan®No. JCZ18109『

\section{Availability of data and materials}

Data sharing not applicable to this article as no data-sets were generated or analysed during the current study.

\section{Ethics approval and consent to participate}

We obtained the signed informed consent from all patients. The Ethics Committee of the Afliated Hospital of Nantong University approved the use of these specimens and data for our research. 
Not applicable.

Competing interests

The authors declare no conflicts of interest.

\section{References}

1. Jemal A, Tiwari RC, Murray T, Ghafoor A, Samuels A, Ward E, Feuer EJ, Thun MJ, American Cancer S: Cancer statistics, 2004. CA Cancer J Clin 2004, 54(1):8-29.

2. Siegel RL, Miller KD, Jemal A: Cancer statistics, 2019. CA Cancer J Clin 2019, 69(1):7-34.

3. Imielinski M, Berger AH, Hammerman PS, Hernandez B, Pugh TJ, Hodis E, Cho J, Suh J, Capelletti M, Sivachenko A et al: Mapping the hallmarks of lung adenocarcinoma with massively parallel sequencing. Cell 2012, 150(6):1107-1120.

4. Morgensztern $\mathrm{D}, \mathrm{Ng} \mathrm{SH}$, Gao F, Govindan R: Trends in stage distribution for patients with non-small cell lung cancer: a National Cancer Database survey. J Thorac Oncol 2010, 5(1):29-33.

5. Devarakonda S, Morgensztern D, Govindan R: Genomic alterations in lung adenocarcinoma. The Lancet Oncology 2015, 16(7):e342-e351.

6. Christiansen JJ, Rajasekaran AK: Reassessing epithelial to mesenchymal transition as a prerequisite for carcinoma invasion and metastasis. Cancer Res 2006, 66(17):8319-8326.

7. Thiery JP: Epithelial-mesenchymal transitions in tumour progression. Nat Rev Cancer 2002, 2(6):442454.

8. Kalluri R, Weinberg RA: The basics of epithelial-mesenchymal transition. J Clin Invest 2009, 119(6):1420-1428.

9. Fidler IJ: The pathogenesis of cancer metastasis: the 'seed and soil' hypothesis revisited. Nat Rev Cancer 2003, 3(6):453-458.

10. van Staalduinen J, Baker D, Ten Dijke P, van Dam H: Epithelial-mesenchymal-transition-inducing transcription factors: new targets for tackling chemoresistance in cancer? Oncogene 2018, 37(48):6195-6211.

11. Oda H, Tsukita S, Takeichi M: Dynamic behavior of the cadherin-based cell-cell adhesion system during Drosophila gastrulation. Dev Bio/ 1998, 203(2):435-450.

12. Miyoshi A, Kitajima Y, Sumi K, Sato K, Hagiwara A, Koga Y, Miyazaki K: Snail and SIP1 increase cancer invasion by upregulating MMP family in hepatocellular carcinoma cells. Br J Cancer 2004, 90(6):1265-1273.

13. Pan S, Hu Y, Hu M, Jian H, Chen M, Gan L, Zheng P, He Y, Wang J: Platelet-derived PDGF promotes the invasion and metastasis of cholangiocarcinoma by upregulating MMP2/MMP9 expression and inducing EMT via the p38/MAPK signalling pathway. Am J Trans/ Res 2020, 12(7):3577-3595.

14. Wu DM, Deng SH, Liu T, Han R, Zhang T, Xu Y: TGF-beta-mediated exosomal Inc-MMP2-2 regulates migration and invasion of lung cancer cells to the vasculature by promoting MMP2 expression. 
Cancer Med 2018, 7(10):5118-5129.

15. Otsuki Y, Saya H, Arima Y: Prospects for new lung cancer treatments that target EMT signaling. Dev Dyn 2018, 247(3):462-472.

16. Kerscher O, Felberbaum R, Hochstrasser M: Modification of proteins by ubiquitin and ubiquitin-like proteins. Annu Rev Cell Dev Biol 2006, 22:159-180.

17. Broomfield $S$, Chow BL, Xiao W: MMS2, encoding a ubiquitin-conjugating-enzyme-like protein, is a member of the yeast error-free postreplication repair pathway. Proc Natl Acad Sci U S A 1998, 95(10):5678-5683.

18. Hofmann RM, Pickart CM: Noncanonical MMS2-encoded ubiquitin-conjugating enzyme functions in assembly of novel polyubiquitin chains for DNA repair. Cell 1999, 96(5):645-653.

19. Andersen PL, Zhou H, Pastushok L, Moraes T, McKenna S, Ziola B, Ellison MJ, Dixit VM, Xiao W: Distinct regulation of Ubc13 functions by the two ubiquitin-conjugating enzyme variants Mms2 and Uev1A. J Cell Biol 2005, 170(5):745-755.

20. Dikshit A, Jin YJ, Degan S, Hwang J, Foster MW, Li CY, Zhang JY: UBE2N Promotes Melanoma Growth via MEK/FRA1/SOX10 Signaling. Cancer Res 2018, 78(22):6462-6472.

21. Zhang L, Sui Y, Wang T, Li L, Li Y, Jin C, Xu F: [Roles of hMMS2 gene in reversing the oxaliplatin tolerance of human colon carcinoma cells]. Yi Chuan 2014, 36(4):346-353.

22. Santarpia L, Iwamoto T, Di Leo A, Hayashi N, Bottai G, Stampfer M, Andre F, Turner NC, Symmans WF, Hortobagyi GN et al: DNA repair gene patterns as prognostic and predictive factors in molecular breast cancer subtypes. Oncologist 2013, 18(10):1063-1073.

23. Subramanian A, Tamayo P, Mootha VK, Mukherjee S, Ebert BL, Gillette MA, Paulovich A, Pomeroy SL, Golub TR, Lander ES et al: Gene set enrichment analysis: a knowledge-based approach for interpreting genome-wide expression profiles. Proc Natl Acad Sci U S A 2005, 102(43):15545-15550.

24. Wiener R, Zhang X, Wang T, Wolberger C: The mechanism of OTUB1-mediated inhibition of ubiquitination. Nature 2012, 483(7391):618-622.

25. Xiao W, Chow BL, Fontanie T, Ma L, Bacchetti S, Hryciw T, Broomfield S: Genetic interactions between error-prone and error-free postreplication repair pathways in Saccharomyces cerevisiae. Mutat Res 1999, 435(1):1-11.

26. Shen T, Cai LD, Liu YH, Li S, Gan WJ, Li XM, Wang JR, Guo PD, Zhou Q, Lu XX et al: Ube2v1-mediated ubiquitination and degradation of Sirt1 promotes metastasis of colorectal cancer by epigenetically suppressing autophagy. J Hematol Oncol 2018, 11(1):95.

27. Meng F, Wu G: The rejuvenated scenario of epithelial-mesenchymal transition (EMT) and cancer metastasis. Cancer Metastasis Rev 2012, 31(3-4):455-467.

28. O'Leary K, Shia A, Schmid P: Epigenetic Regulation of EMT in Non-Small Cell Lung Cancer. Curr Cancer Drug Targets 2018, 18(1):89-96.

29. Zhu X, Chen L, Liu L, Niu X: EMT-Mediated Acquired EGFR-TKI Resistance in NSCLC: Mechanisms and Strategies. Front Oncol 2019, 9:1044. 
30. VanDemark AP, Hofmann RM, Tsui C, Pickart CM, Wolberger C: Molecular insights into polyubiquitin chain assembly: crystal structure of the Mms2/Ubc13 heterodimer. Cell 2001, 105(6):711-720.

31. Ulrich HD, Jentsch S: Two RING finger proteins mediate cooperation between ubiquitin-conjugating enzymes in DNA repair. EMBO J 2000, 19(13):3388-3397.

32. Bailly V, Lamb J, Sung P, Prakash S, Prakash L: Specific complex formation between yeast RAD6 and RAD18 proteins: a potential mechanism for targeting RAD6 ubiquitin-conjugating activity to DNA damage sites. Genes Dev 1994, 8(7):811-820.

33. Maga G, Hubscher U: Proliferating cell nuclear antigen (PCNA): a dancer with many partners. J Cell Sci 2003, 116(Pt 15):3051-3060.

34. Karras GI, Jentsch S: The RAD6 DNA damage tolerance pathway operates uncoupled from the replication fork and is functional beyond S phase. Cell 2010, 141(2):255-267.

35. Chiu RK, Brun J, Ramaekers C, Theys J, Weng L, Lambin P, Gray DA, Wouters BG: Lysine 63polyubiquitination guards against translesion synthesis-induced mutations. PLoS Genet 2006, 2(7):e116.

36. Gazy I, Kupiec M: The importance of being modified: PCNA modification and DNA damage response. Cell Cycle 2012, 11(14):2620-2623.

37. Haracska L, Torres-Ramos CA, Johnson RE, Prakash S, Prakash L: Opposing effects of ubiquitin conjugation and SUMO modification of PCNA on replicational bypass of DNA lesions in Saccharomyces cerevisiae. Mol Cell Biol 2004, 24(10):4267-4274.

38. Suzuki K, Kachala SS, Kadota K, Shen R, Mo Q, Beer DG, Rusch VW, Travis WD, Adusumilli PS:

Prognostic immune markers in non-small cell lung cancer. Clin Cancer Res 2011, 17(16):5247-5256.

39. Daniel D, Chiu C, Giraudo E, Inoue M, Mizzen LA, Chu NR, Hanahan D: CD4+ T cell-mediated antigenspecific immunotherapy in a mouse model of cervical cancer. Cancer Res 2005, 65(5):2018-2025.

40. Lundin KU, Screpanti V, Omholt H, Hofgaard PO, Yagita H, Grandien A, Bogen B: CD4+ T cells kill Id+ B-lymphoma cells: FasLigand-Fas interaction is dominant in vitro but is redundant in vivo. Cancer Immunol Immunother 2004, 53(12):1135-1145.

41. Dudley ME, Wunderlich J, Nishimura MI, Yu D, Yang JC, Topalian SL, Schwartzentruber DJ, Hwu P, Marincola FM, Sherry R et al: Adoptive transfer of cloned melanoma-reactive T lymphocytes for the treatment of patients with metastatic melanoma. J Immunother 2001, 24(4):363-373.

42. Wang RF: The role of MHC class II-restricted tumor antigens and CD4+ T cells in antitumor immunity. Trends Immunol 2001, 22(5):269-276.

\section{Tables}

Table 1. Association between UBE2V2 expression and pathological parameters of LUAD in the Immunohistochemistry 


\begin{tabular}{|c|c|c|c|c|}
\hline \multirow[t]{2}{*}{ Clinical parameters } & \multirow[t]{2}{*}{ All cases } & \multicolumn{2}{|c|}{ UBE2V2 expression } & \multirow[b]{2}{*}{ P-value } \\
\hline & & Low & High & \\
\hline \multicolumn{5}{|l|}{ age } \\
\hline$\leq 65$ & 58 & 28 & 30 & 0.413 \\
\hline$>65$ & 33 & 13 & 20 & \\
\hline \multicolumn{5}{|l|}{ Gender } \\
\hline Male & 59 & 22 & 37 & 0.021 \\
\hline Female & 32 & 20 & 12 & \\
\hline \multicolumn{5}{|l|}{ Smoking } \\
\hline Nonsmoker & 71 & 35 & 36 & 0.215 \\
\hline Smoker & 20 & 8 & 12 & \\
\hline \multicolumn{5}{|l|}{ Stage } \\
\hline I & 47 & 26 & 21 & 0.042 \\
\hline $11+\square+\square$ & 44 & 15 & 29 & \\
\hline \multicolumn{5}{|l|}{ T classification } \\
\hline $\mathrm{T} 1+\mathrm{T} 2$ & 85 & 38 & 47 & 0.563 \\
\hline $\mathrm{T} 3+\mathrm{T} 4$ & 6 & 3 & 3 & \\
\hline \multicolumn{5}{|c|}{ Distant metastasis status } \\
\hline MO & 87 & 39 & 48 & 0.613 \\
\hline M1 & 4 & 2 & 2 & \\
\hline \multicolumn{5}{|c|}{ Lymph node metastasis } \\
\hline Yes & 57 & 31 & 26 & 0.021 \\
\hline No & 34 & 10 & 24 & \\
\hline \multicolumn{5}{|l|}{ degree } \\
\hline Low grade & 27 & 18 & 9 & 0.015 \\
\hline Middle grade & 61 & 40 & 21 & \\
\hline High grade & 3 & 1 & 2 & \\
\hline
\end{tabular}


$\mathrm{P}<0.05$ was considered to indicate a statistically significant difference.

Table 2. The relationship between UBE2V2 expression and overall survival in patients with lung cancer was analyzed by Univariate and multivariate analysis in the TCGA.

\begin{tabular}{|lllllll|}
\hline \multirow{2}{*}{ Parameter } & \multicolumn{3}{l}{ Univariate analysis } & \multicolumn{4}{l|}{ Multivariate analysis } \\
\cline { 2 - 7 } & HR & $95 \% \mathrm{Cl}$ & P-value & HR & $95 \% \mathrm{Cl}$ & P-value \\
\hline age & 1.012 & $0.995-1.029$ & 0.145 & 1.018 & $1.002-1.036$ & $\mathbf{0 . 0 2 5}$ \\
\hline gender & 1.084 & $0.787-1.493$ & 0.619 & 0.903 & $0.649-1.258$ & 0.054 \\
\hline stage & 1.655 & $1.426-1.921$ & $\mathbf{3 . 0 7 E}-11$ & 1.474 & $1.184-1.835$ & $\mathbf{0 . 0 0 0 5}$ \\
\hline T & 1.535 & $1.259-1.872$ & $\mathbf{2 . 2 4 E}-05$ & 1.122 & $0.905-1.391$ & 0.292 \\
\hline M & - & - & - & - & - & - \\
\hline N & 1.699 & $1.419-2.033$ & $\mathbf{7 . 1 7 E}-09$ & 1.213 & $0.949-1.551$ & 0.121 \\
\hline UBE2V2 & 1.469 & $1.078-2.002$ & $\mathbf{0 . 0 1 4}$ & 1.497 & $1.073-2.089$ & $\mathbf{0 . 0 1 2}$ \\
\hline
\end{tabular}

$\mathrm{P}<0.05$ was considered to indicate a statistically significant difference.

Table 3. The relationship between UBE2V2 expression and overall survival in patients with lung cancer was analyzed by Univariate and multivariate analysis in the Immunohistochemistry 


\begin{tabular}{|c|c|c|c|c|c|c|}
\hline \multirow[t]{2}{*}{ Parameter } & \multicolumn{3}{|c|}{ Univariate analysis } & \multicolumn{3}{|c|}{ Multivariate analysis } \\
\hline & $H R$ & $95 \% \mathrm{Cl}$ & P-value & $\mathrm{HR}$ & $95 \% \mathrm{Cl}$ & P-value \\
\hline UBE2V2 & 2.002 & $1.139-3.517$ & 0.016 & 1.842 & $1.022-3.317$ & 0.042 \\
\hline \multicolumn{7}{|l|}{ high vs low } \\
\hline Age (years) & 1.345 & $0.786-2.303$ & 0.279 & & & \\
\hline \multicolumn{7}{|l|}{$\leq 65$ vs $>65$} \\
\hline Gender & 0.678 & $0.378-1.128$ & 0.194 & & & \\
\hline \multicolumn{7}{|l|}{ male vs. female } \\
\hline T classification & 1.157 & $0.831-1.611$ & 0.388 & & & \\
\hline \multicolumn{7}{|c|}{$\mathrm{T} 1$ vs $\mathrm{T} 2$ vs $\mathrm{T} 3+\mathrm{T} 4$} \\
\hline M classification & 0.621 & $0.151-2.551$ & 0.508 & & & \\
\hline \multicolumn{7}{|l|}{ yes vs no } \\
\hline Different degree & 0.306 & $0.460-1.276$ & 0.766 & & & \\
\hline \multicolumn{7}{|c|}{ low vs middle vs high } \\
\hline TMN stage & 1.127 & $0.634-2.299$ & 0.411 & & & \\
\hline$\llbracket v s . \otimes v s . \rrbracket+\rrbracket$ & & & & & & \\
\hline
\end{tabular}

$\mathrm{P}<0.05$ was considered to indicate a statistically significant difference.

\section{Figures}


(a)
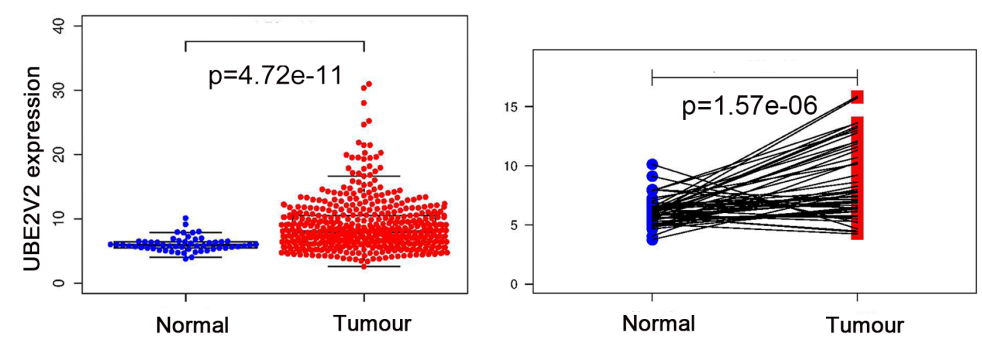

(c)

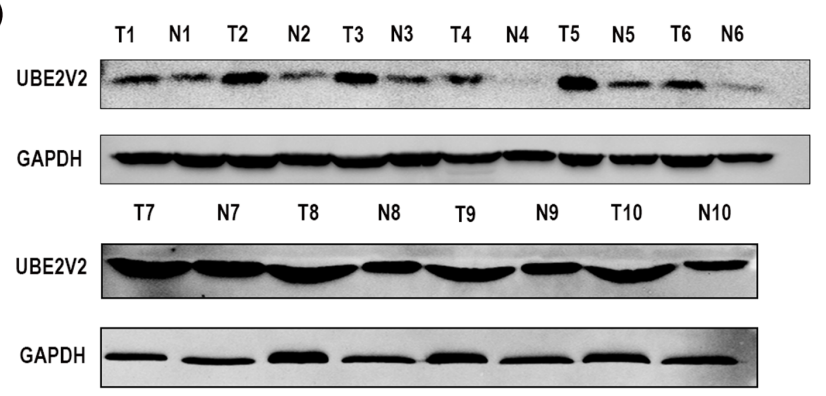

(d)

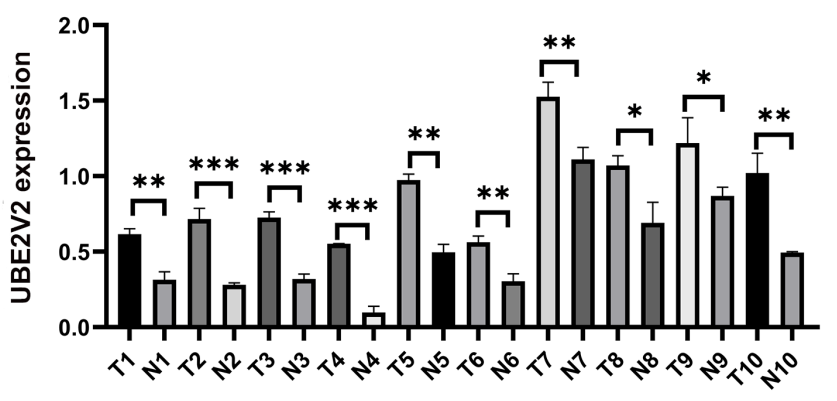

(e)

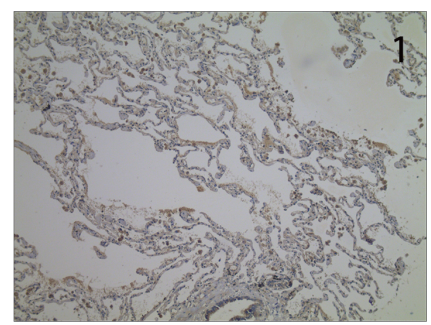

(f)

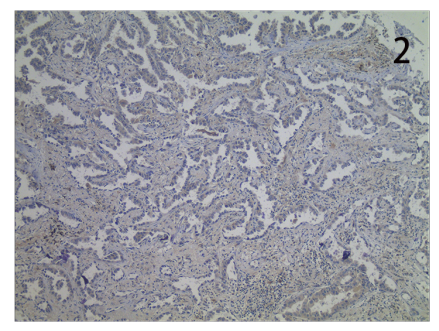

(g)

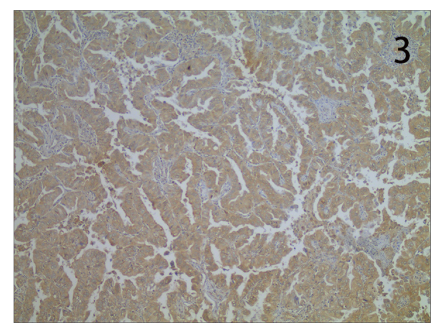

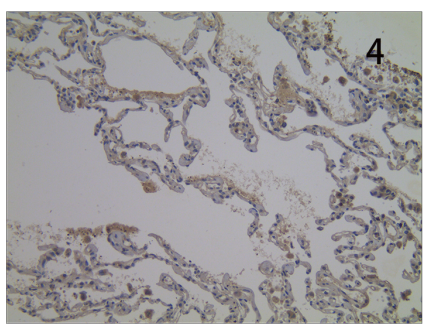
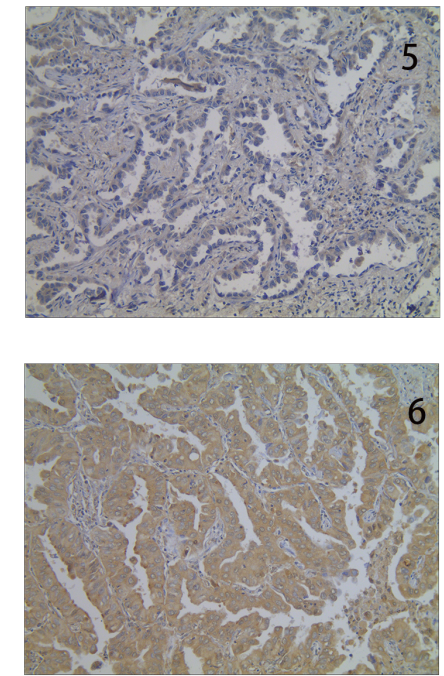

Figure 1

UBE2V2 expression in LUAD and normal tissues. (a)The mRNA expression of UBE2V2 between 497 LUAD tissues and 54 normal samples from TCGA囚red dots represent tumour samples and the blue dots represent normal samples $\rrbracket$. (b) The mRNA expression of UBE2V2 in 54 LUAD samples and its matched vicinal samples. (c) Western blotting revealed the upregulation of UBE2V2 expression in 10 pairs fresh LUAD samples(T). (d) We used ImageJ software to measure the band density of UBE2V2 protein. (e) Negative expression of UBE2V2 protein in adjacent normal tissues. (f)Negative expression of UBE2V2 protein in lung adenocarcinoma tissue. (g)Positive expression of UBE2V2 protein in lung adenocarcinoma tissue (a1,b2,c3 $\times 200$ magnification; a4,b5,c6 $\times 400$ magnification). ${ }^{*} P<0.05 ;{ }^{\star *} P<0.01 ;{ }^{* \star \star} P<0.001$. (UBE2V2: Ubiquitin-conjugating E2 enzyme variants 2. TCGA: The Cancer Genome Atlas. LUAD: lung adenocarcinoma.) 

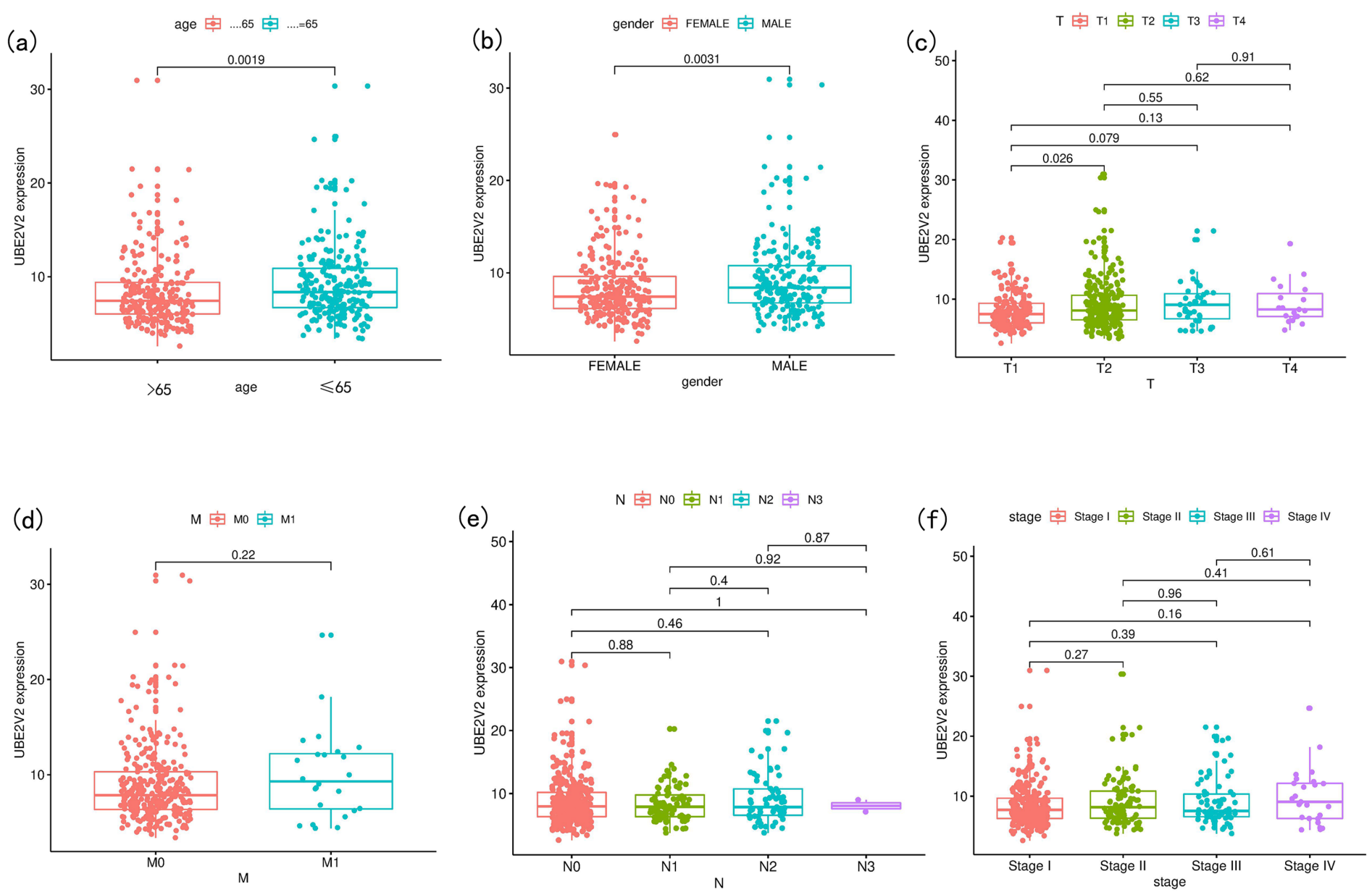

\section{Figure 2}

Association of UBE2V2 expression with clinical parameters based on the TCGA. (a) age, (b)gender, (c) tumor stage, (d) distant metastases (e) lymph node metastasis, $(f)$ clinical stage 
(a)

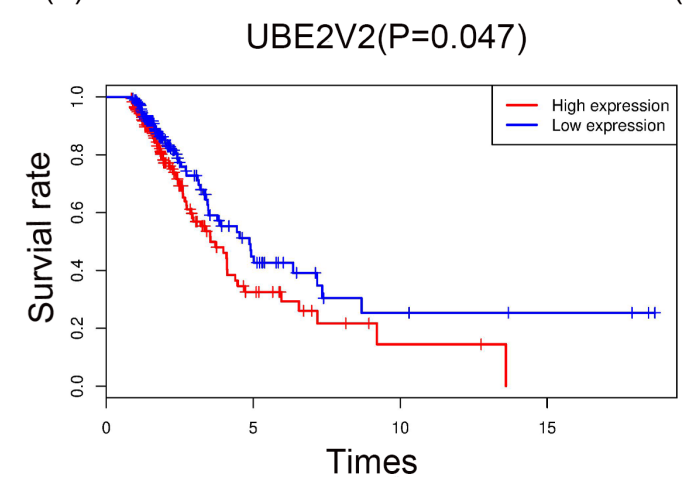

(d)

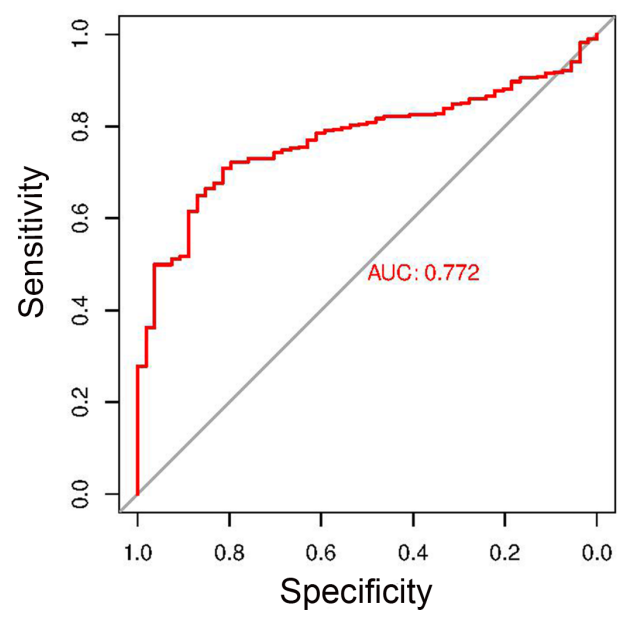

(b)

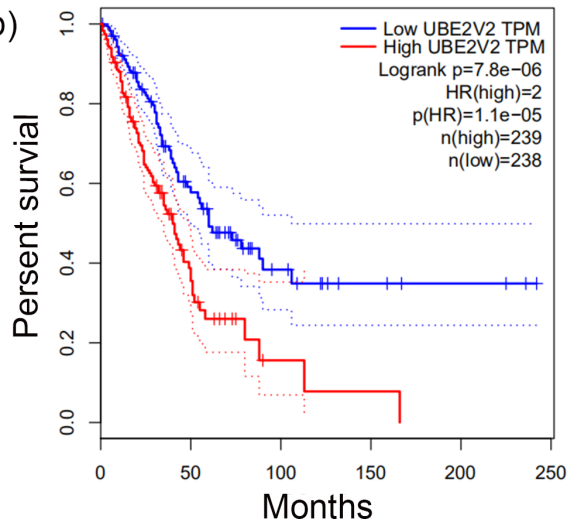

(e)

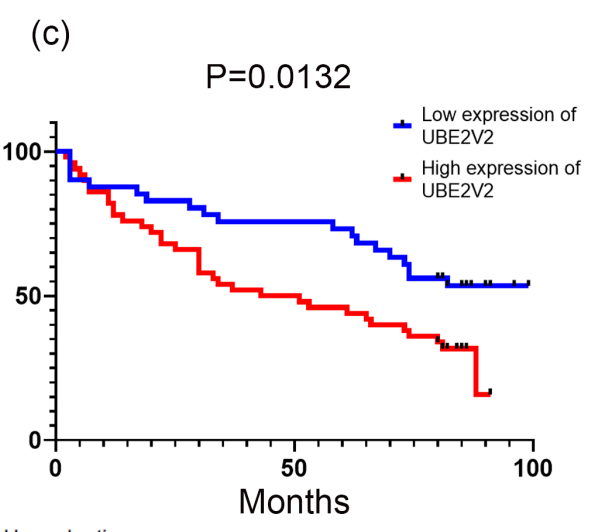

Hazard ratio

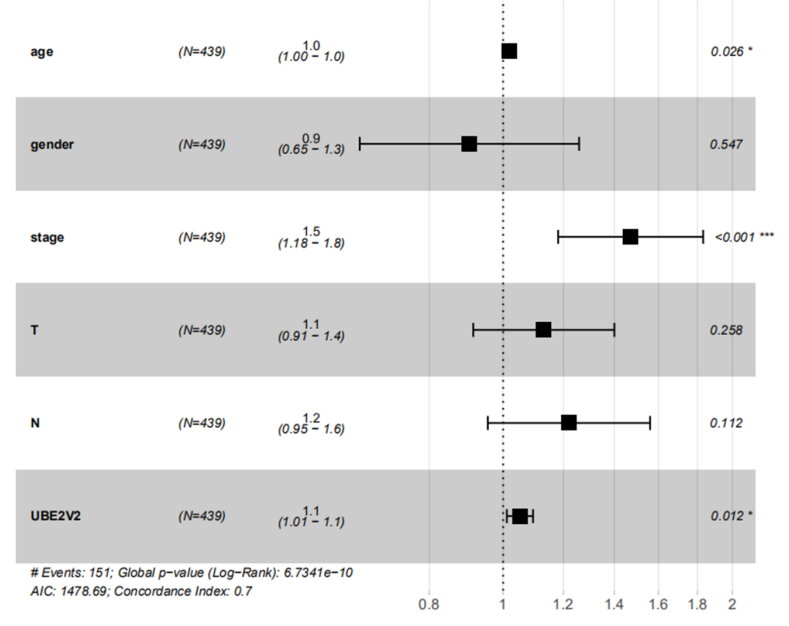

\section{Figure 3}

The expression level of UBE2V2 was bound up with the overall survival in patients. (a) Survival curve of UBE2V2 expression based on TCGA database in LUAD patients. (b囚Overall Survival in GEPIA.(c) Percent Survival of UBE2V2 in 91 pairs LUAD tissues from ICH (the red curve represents high expression of UBE2V2, and the blue curve represents low expression of UBE2V2). $\triangle \mathrm{d} \nabla$ Receiver operating characteristic (ROC) curve for UBE2V2 expression in 497 LUAD patients and 54 healthy individuals. (e) UBE2V2 was identified as an independent prognostic factor by multivariate analysis. 
(a)

(b)

\section{H1299 SPCA1 A549 H1650}

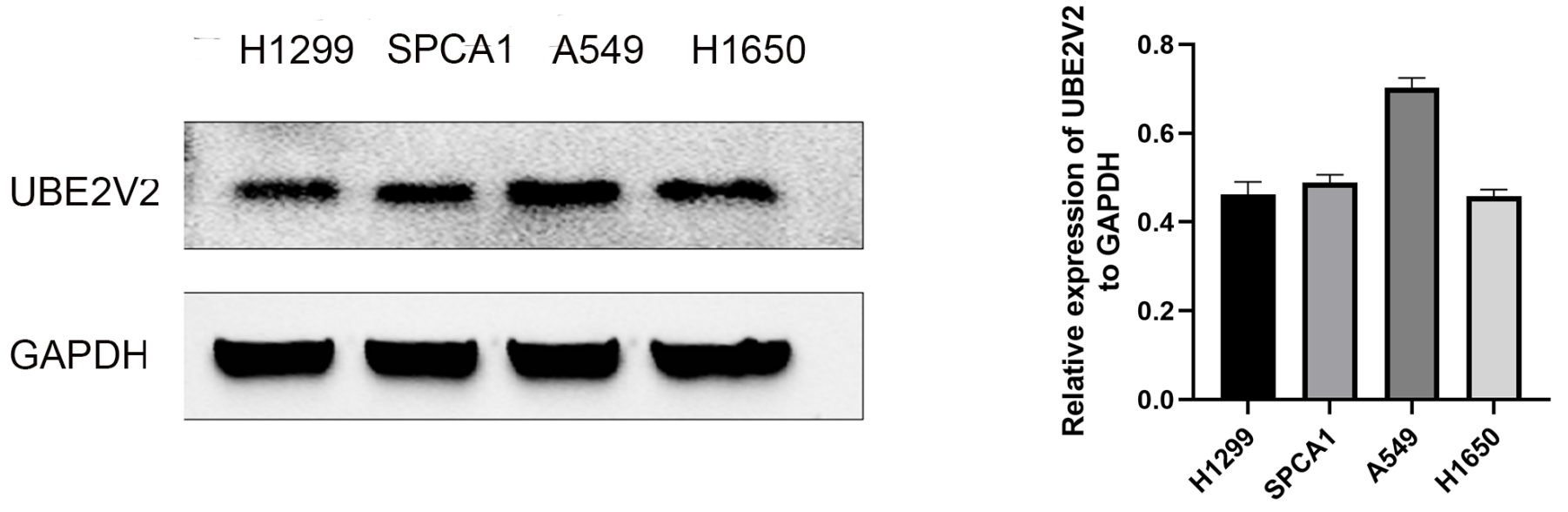

(c)

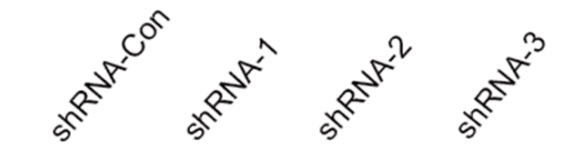

PCNA

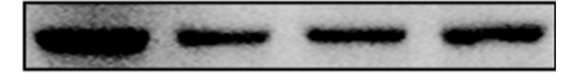

GAPDH

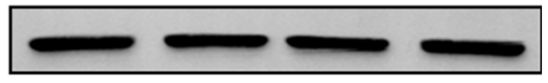

A549

PCNA

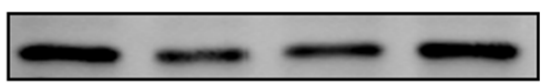

GAPDH

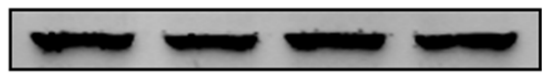

SPCA1 (d)

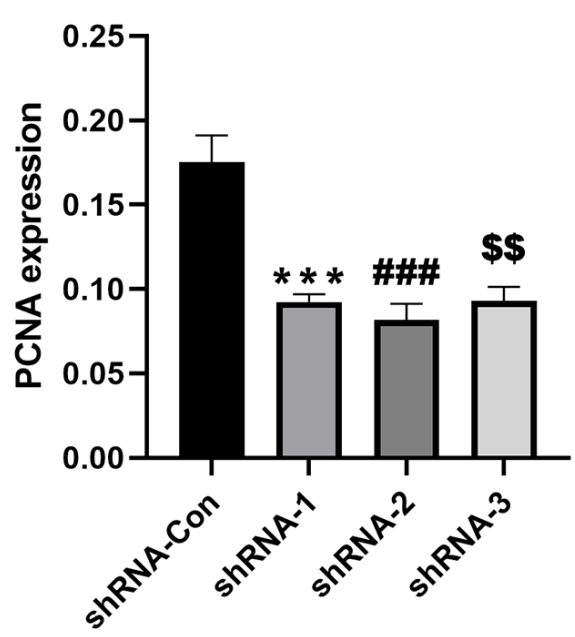

A549 (e)

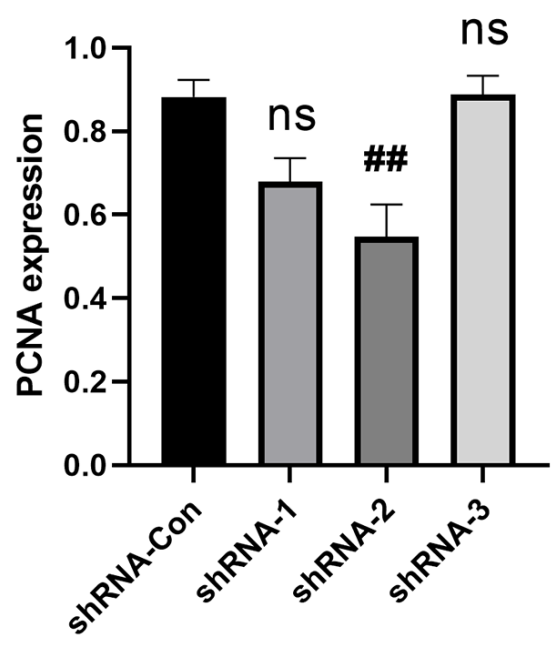

SPCA1

\section{Figure 4}

UBE2V2 protein expression levels in different LUAD cell lines and UBE2V2 effected the proliferation marker PCNA. (a) The expression of UBE2V2 in SPCA1, A549, H1299 and H1650 cells. (b) The histogram by optical density method showed that UBE2V2 has high expression levels in A549 and SPCA1 cells. (c-e) Knockdown of UBE2V2 (especially shRNA-2) obviously reduced the expression of PCNA. ${ }^{\star *} P<0.01$, $\star \star \star P<0.001 ; \# \# \# P<0.001 ; \$ \$ P<0.01 ; n s \mathrm{P} \otimes 0.05$. 


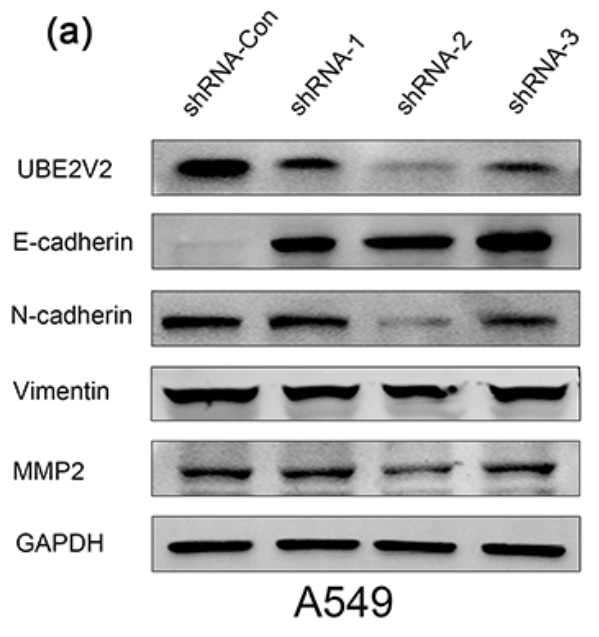

(d)

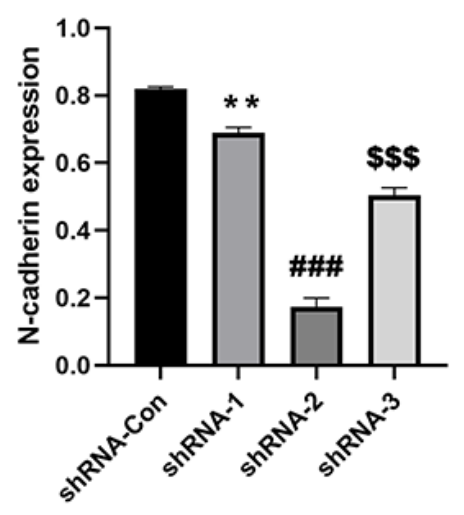

(b)

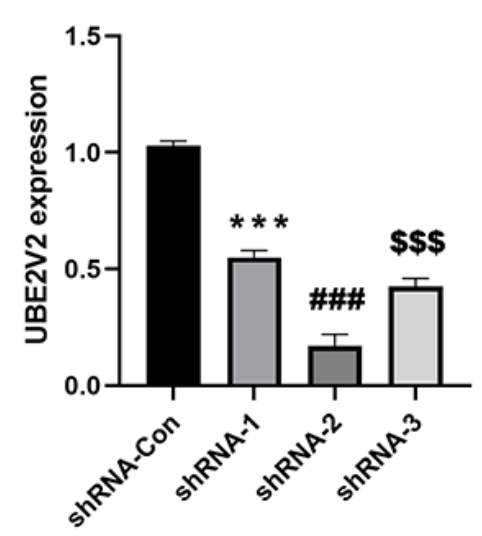

(e)

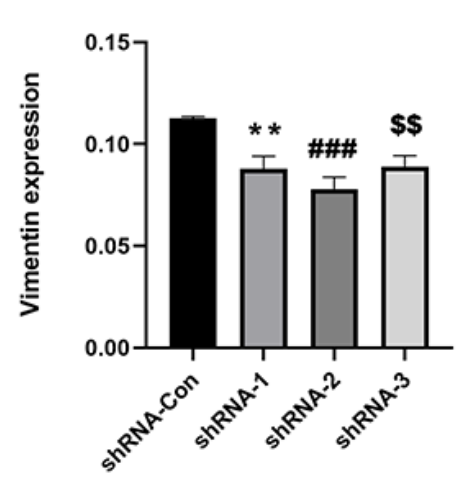

(c)

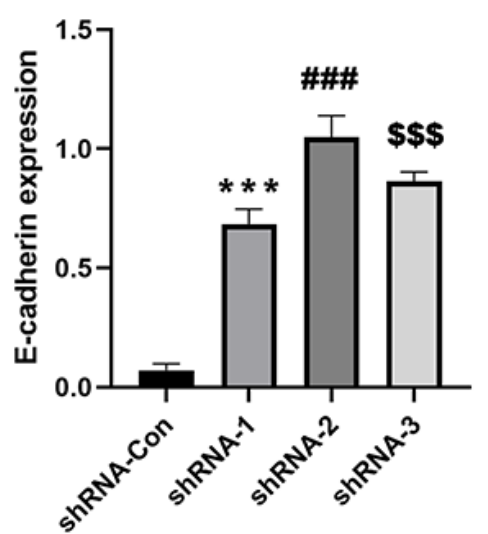

(f)

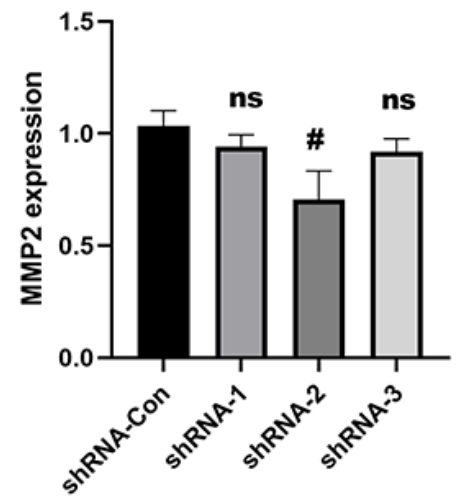

(g)
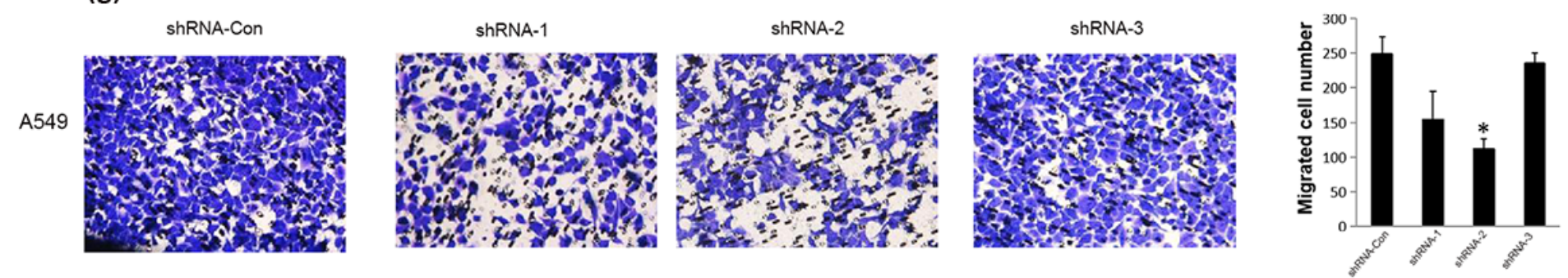

Figure 5

Knockdown UBE2V2 inhibited migration and invasion in A549 cells. (a) Western blotting revealed that knockdown of UBE2V2(especially shRNA-2) upregulated E-cadherin while decreased Vimentin, N-cadherin and MMP2 in A549 cells. (b-f) Data analysis of (a). (g) Transwell analysis showed that the knockdown UBE2V2 (shRNA-2) significantly reduced migration than the control group in A549 cells. ${ }^{\star} P<0.05,{ }^{\star \star} P<0.01$, $\star \star \star P<0.001 ; \# P<0.05, \# \# \# P<0.001$;

$$
P<0.01,
$$

$\$ \mathrm{P}<0.001 ; n s \mathrm{P} \llbracket 0.05$. 


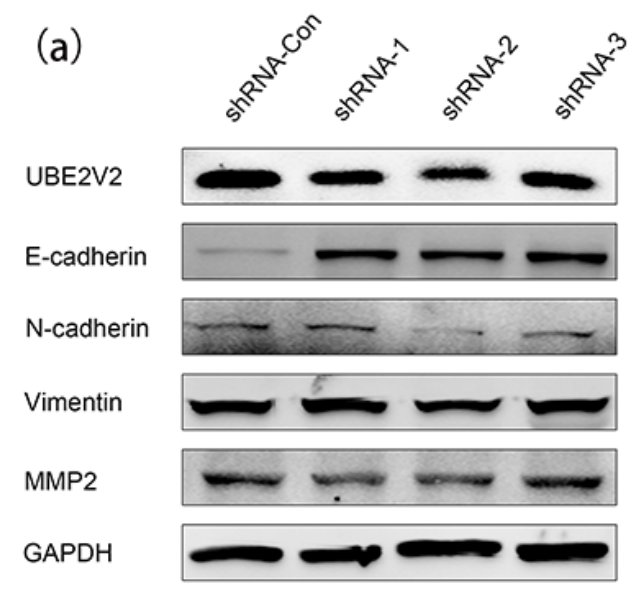

SPCA1

(d)

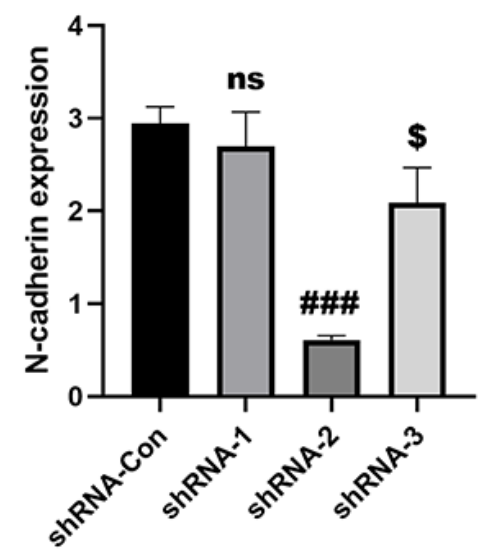

(g)
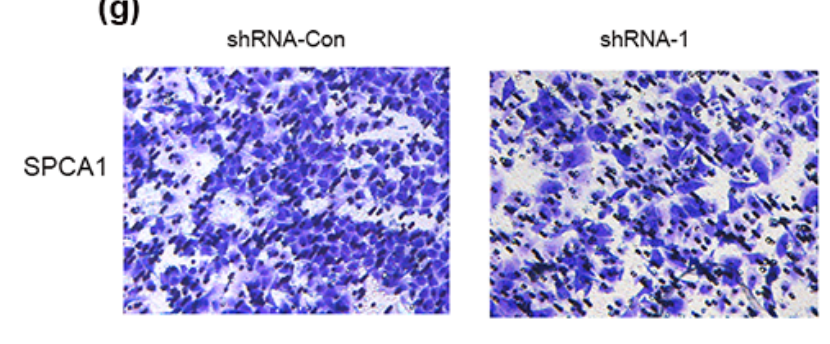

(b)

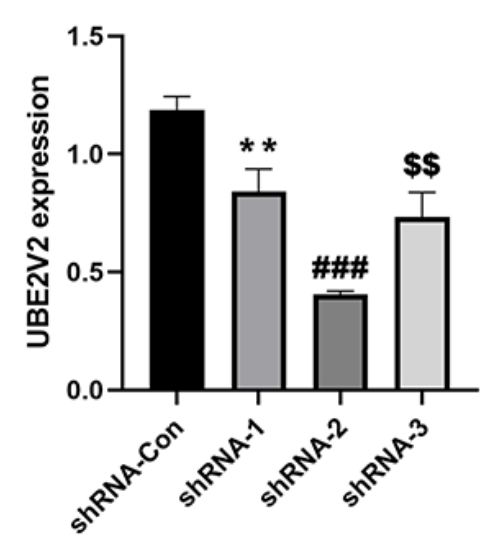

(e)

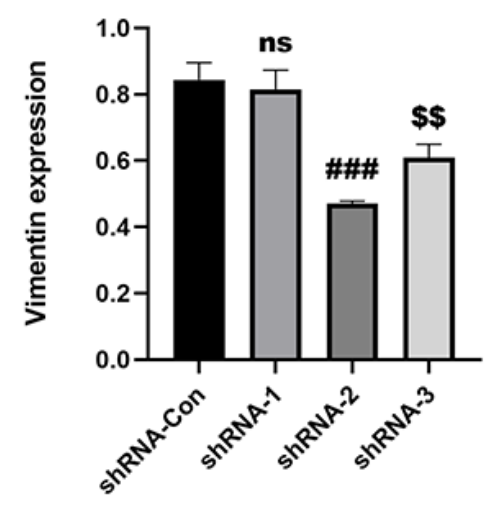

(c)

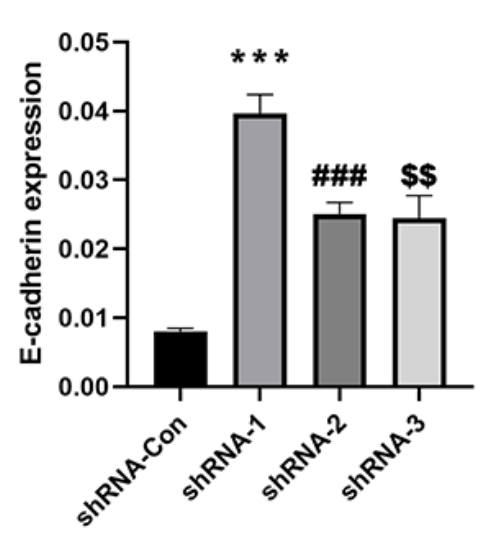

(f)

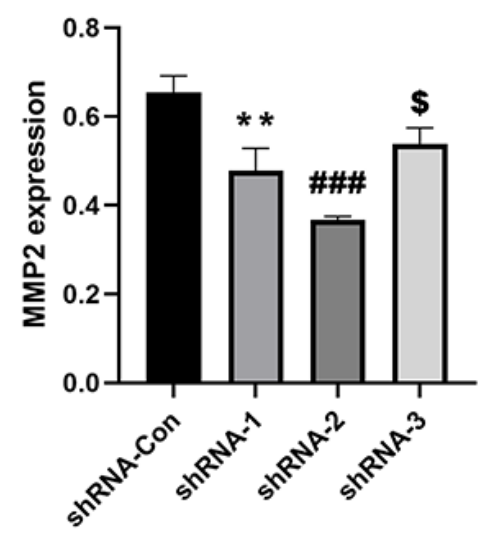

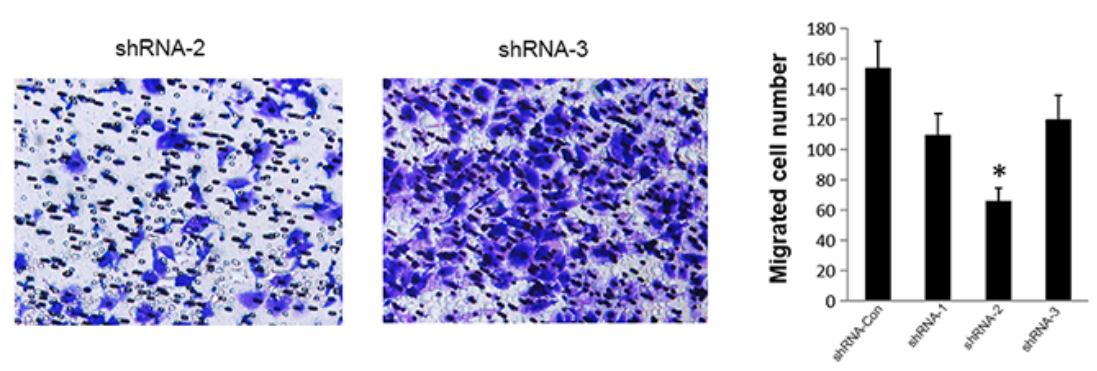

Figure 6

Knockdown of UBE2V2 suppressed migration and invasion in SPCA1 cells. (a) Western blotting revealed that knockdown of UBE2V2(especially shRNA-2) upregulated E-cadherin and decreased Vimentin, $\mathrm{N}$ cadherin and MMP2 in SPCA1 cells. (b-f) Data analysis of (a). (g) Transwell analysis showed that the knockdown UBE2V2 (shRNA-2) significantly reduced migration than the control group in SPCA1 cells. $\star * P<0.01,{ }^{* \star *} P<0.001 ; \# \# \# P<0.001 ; \$ P<0.05, \$ \$ P<0.01 ; n s P \otimes 0.05$. 

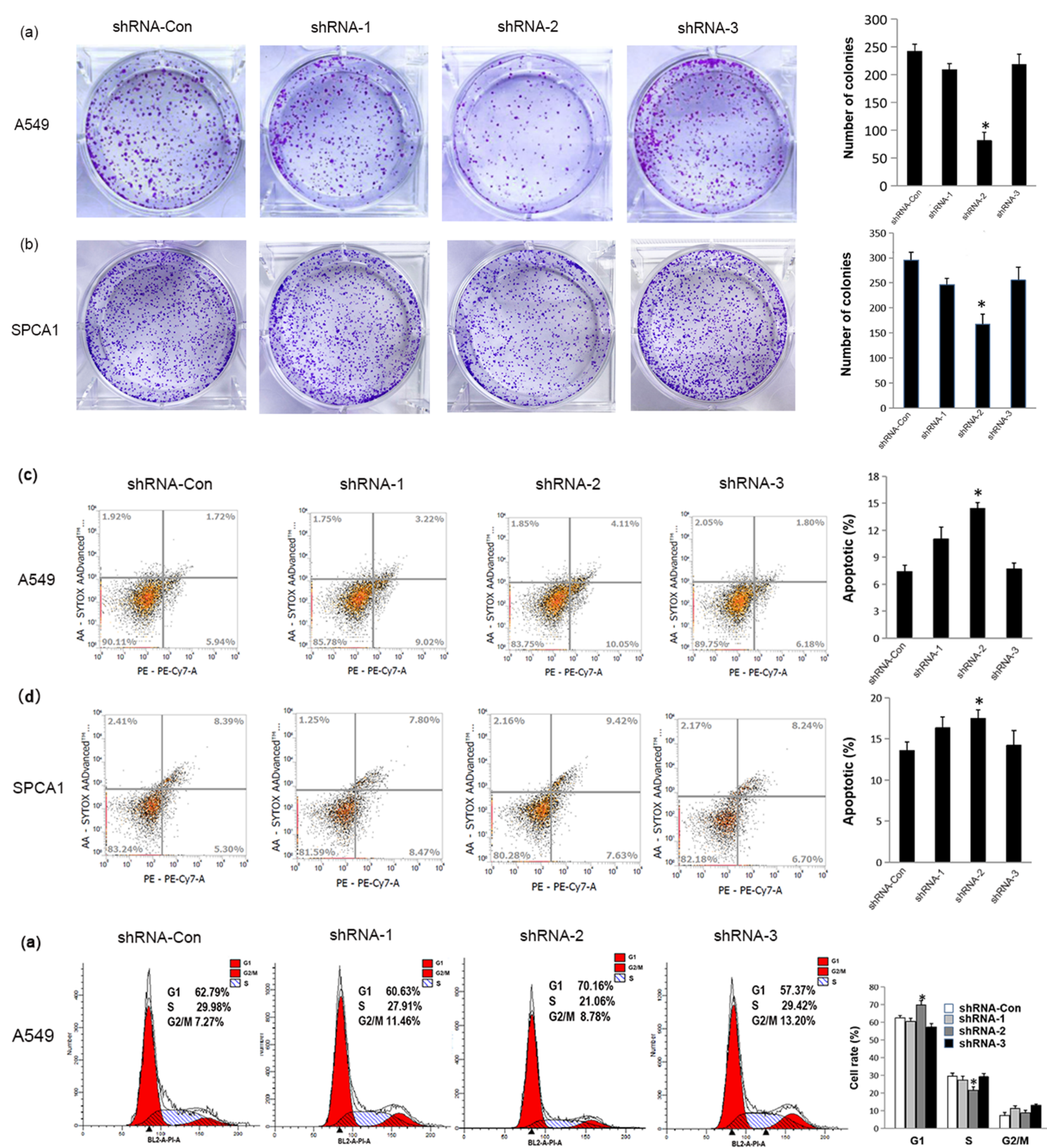

shRNA-3

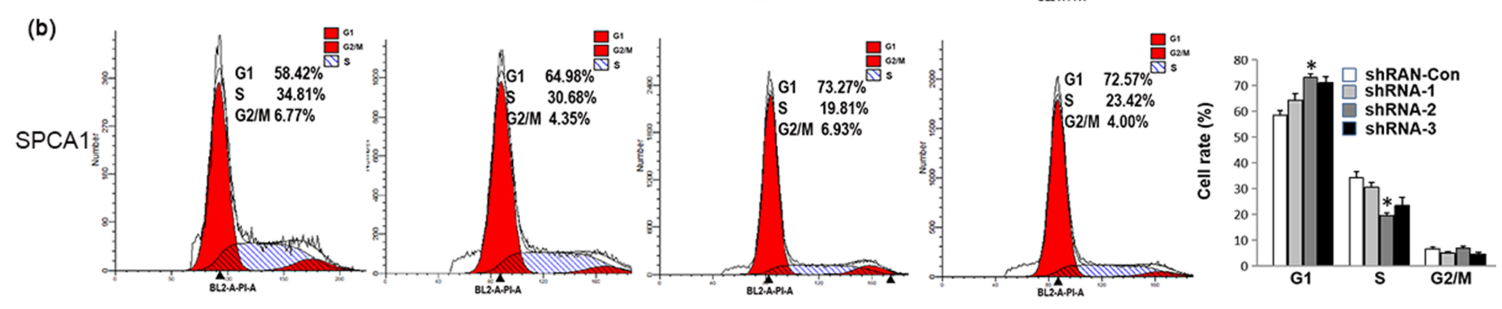

\section{Figure 7}

Colony formation assay, Apoptosis analysis and Cell cycle test. $₫ a, b \triangle K$ nockdown of UBE2V2 (especially shRNA-2) reduced colony the number of cell clones compared to shCon. (c, d) Knockdown of UBE2V2 (especially shRNA-2) significantly increased the number of apoptotic cells compared to shCon. $(e, f)$ Knockdown of UBE2V2 (especially shRNA-2) increased the proportion of cells in the GI phase and remarkably diminished the proportion of cells in the DNA synthesis phase (S phase). ${ }^{*} \mathbb{P} \otimes 0.05$. 
(a)

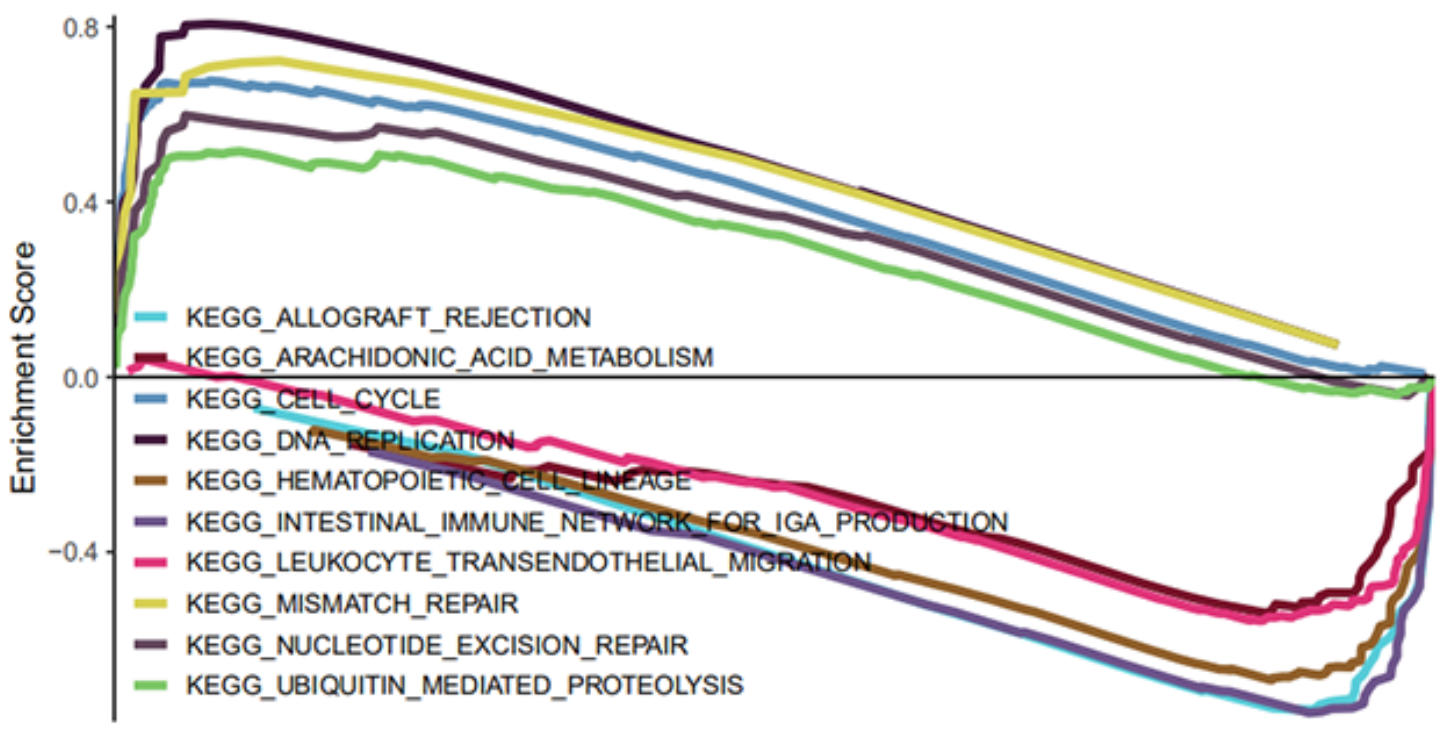

(b)

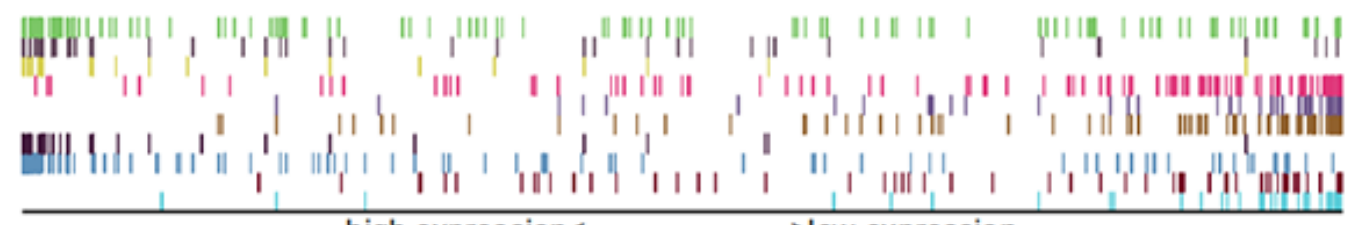

high expression<------------>low expression
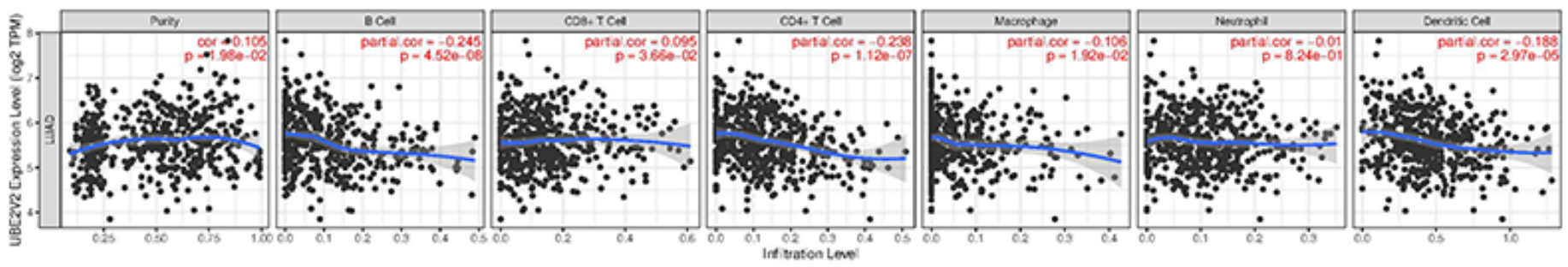

Figure 8

(a)The gene set enrichment analysis (GSEA) determined UBE2V2 correlated signal pathways. (b) The relationship between UBE2V2 expression and different immune infiltrating cells. 